\title{
SCHATTEN $p$-NORM INEQUALITIES RELATED TO A CHARACTERIZATION OF INNER PRODUCT SPACES
}

\section{Omar Hirzallah, Fuad Kittaneh and Mohammad Sal Moslehian}

Abstract. Let $A_{1}, \cdots A_{n}$ be operators acting on a separable complex Hilbert space such that $\sum_{i=1}^{n} A_{i}=0$. It is shown that if $A_{1}, \cdots A_{n}$ belong to a Schatten $p$-class, for some $p>0$, then

$$
2^{p / 2} n^{p-1} \sum_{i=1}^{n}\left\|A_{i}\right\|_{p}^{p} \leqslant \sum_{i, j=1}^{n}\left\|A_{i} \pm A_{j}\right\|_{p}^{p}
$$

for $0<p \leqslant 2$, and the reverse inequality holds for $2 \leqslant p<\infty$. Moreover,

$$
\sum_{i, j=1}^{n}\left\|A_{i} \pm A_{j}\right\|_{p}^{2} \leqslant 2 n^{2 / p} \sum_{i=1}^{n}\left\|A_{i}\right\|_{p}^{2}
$$

for $0<p \leqslant 2$, and the reverse inequality holds for $2 \leqslant p<\infty$. These inequalities are related to a characterization of inner product spaces due to E.R. Lorch.

Mathematics subject classification (2010): Primary: 47A30; Secondary: 46C15, 47B10, 47B15. Keywords and phrases: Schatten $p$-norm, positive operator, inequality, inner product space.

\section{REFERENCES}

[1] R. Bhatia, Matrix Analysis, Springer-Verlag, New York, 1997.

[2] R. Bhatia And F. Kittaneh, Cartesian decompositions and Schatten norms, Linear Algebra Appl., 311 (2000), 109-116.

[3] R. Bhatia And F. Kittaneh, Clarkson inequalities with several operators, Bull. London Math. Soc., 36 (2004), 820-832.

[4] F. Kittaneh, Norm inequalities for certain operator sums, J. Funct. Anal., 143 (1997), 337-348.

[5] E. R. LORCH, On certain implications which characterize Hilbert space, Ann. of Math., 249 (1948), $523-532$.

[6] B. Simon, Trace Ideals and their Applications, Cambridge University Press, Cambridge, 1979. 